\title{
Review of: "Climatic niche conservatism shapes the ecological assembly of Hawaiian arthropod communities"
}

\author{
Kadeem Gilbert ${ }^{1}$ \\ 1 Pennsylvania State University
}

Potential competing interests: The author(s) declared that no potential competing interests exist.

This is an excellent study addressing the classic problem of elevational diversity gradients. Metabarcoding is a powerful approach that allows finer-scale whole-community level analyses without some of the impediments that traditional taxonomic approaches present, i.e., requiring many individual experts who each must specialize on particular taxa, on top of the amount of time needed to ID a specimen in cases of ambiguity. Metabarcoding has been notably revolutionary for including the smallest, hardest to identify organisms that have been traditionally excluded from community ecology studies (especially microbes). While the OTU approach is recently being replaced by the ASV approach, the OTU approach still provides the major benefits of metabarcoding (the only disadvantage relative to ASVs being the inability to compare OTUs across studies). The authors complement the data obtained from metabarcoding with specimen counts, which helps overcome the limitations of metabarcoding regarding quantifying abundance (at best, metabarcoding can only give relative abundance of OTUs); this is a great approach, however I do not think that the claim that this approach is novel is entirely accurate, since prior studies have combined specimen counts with barcoding in this way (e.g., Bittleston et al. 2016, Littlefair et al. 2018, Gilbert et al. 2020). Regardless, the rigor of the authors' experimental and statistical design is laudable and a major contribution to understanding of the forces shaping community assembly processes along elevational gradients.

Arthropod communities likely contain many cryptic species which may be difficult or impossible to detect with traditional taxonomic approaches, hence the utility of capturing a fuller picture of the species richness in this study. However, taxonomic assignments that are made on the basis of sequence similarity cutoffs using a single barcode gene still may obscure some of the actual diversity. For instance, the approach says little about functional diversity since organisms that are identical at neutral genetic markers may differ in other regions across their genomes; and even taxa that have very little genotypic divergence may differ in some important ways at the phenotypic level. Sorting these arthropods into size classes, while a very rough proxy for function, was an important addition that allowed at least some discussion of function, although it must be questioned how much can be said about the niches that these particular OTUs occupy, when the only ecological data directly observed are broad taxonomic assignments and size classes. It is 
unclear to me why Collembola were treated as a separate size class-I understand that because the community was disproportionately made up of Collembola the authors wanted to check whether those OTUs might have an outsized effect on the overall patterns observed, but I do not understand why there was not a discussion of what patterns could be seen when analyzing Collembola alone and whether the inclusion of Collembola actually altered the community-level trends in any way. Relatedly, I question why the authors excluded OTUs from orders representing non-native species. Why not analyze native and nonnative taxa separately to see whether "nativeness" influences any of the trends that were observed? Also, can the authors exclude the possibility that some subset of the OTUs in the other orders were also nonnative? Further, if native and non-native species are coexisting along the transect, do they not both equally count as members of the overall arthropod community, given that they interact or at least compete for resources? What justification is there for excluding parts of the community when analyzing communitylevel trends?

The ecological niche modelling is a good way of gaining some rough approximation of the fundamental niche of the study organisms. However, I wonder how well the largescale climate data obtained from satellites reflects the actual realized niche of these arthropods in terms of temperature and humidity, considering that the microhabitats they occupy very likely buffer external temperature in ways that coarse-grained models may not capture. Ideally it would be best to measure abiotic variables at the scale of the organisms in question-that is not always feasible, but I think the coarseness of largescale climate data is a caveat worth mentioning, especially for small-bodied organisms. At least, in this case, the organisms were all collected from a similar vegetation layer and thus presumably experience a similar microclimate as long as they spend significant portions of their lives in such microenvironments (for this reason I agree with the decision to exclude highly vagile arthropods like fast-flying Diptera and Hymenoptera from the analysis, though it is still difficult to infer whether other the OTUs included might not still have behaviors such as digging that would alter the suite of microhabitats they experience relative to other members of the community).

Overall, this was a strong, well thought-out study that leads to interesting conclusions, and paves the way for future work. While there are some inherent methodological limitations which may not have been possible to overcome, I also think there is potential for a few more interesting analyses that can be done with the existing data as well. I look forward to seeing the finalized published version.

Bittleston LS, Baker C, Strominger LB, Pringle A, Pierce NE . 2016. Metabarcoding as a tool for investigating arthropod diversity in Nepenthes pitcher plants. Austral Ecology 41: 120-132.

Littlefair JE, Zander A, de Sena Costa C, Clare EL . 2019. DNA metabarcoding reveals changes in the contents of carnivorous plants along an elevation gradient. Molecular Ecology 28: 281-292. 
Gilbert KJ, Bittleston LS, Naive MAK, Kiszewski AE, Buenavente PAC, Lohman DJ, Pierce NE . 2020. Investigation of an Elevational Gradient Reveals Strong Differences Between Bacterial and Eukaryotic Communities Coinhabiting Nepenthes Phytotelmata. Microbial Ecology: 1-16. 